\title{
Does Consolidation Chemotherapy improve Patient Survival in Locally Advanced Non-Small-Cell Lung Cancer?
}

\author{
Fatma Bugdayci BASAL, Ferit ASLAN, Guliz ZENGIN, Irem BILGETEKIN, \\ Berna OKSUZOGLU, Umut DEMIRCI
}

Dr. Abdurrahman Yurtaslan Oncology Training and Research Hospital, Department of Medical Oncology, Ankara, TURKEY

\begin{abstract}
The role of consolidation chemotherapy is being investigated due to inconclusive outcomes, ascribed to high rates of recurrence and mortality with standard concurrent chemoradiotherapy (CRT) in patients with unresectable locally advanced non-small-cell lung cancer (LA-NSCLC). We evaluated the impact of consolidation chemotherapy on the survival of patients with LA-NSCLC at our single medical centre. In total, 136 patients with unresected LA-NSCLC were evaluated to identify and compare the factors influencing survival rates. The median overall survival (OS) was 27 and 23.6 months and median progression-free survival (PFS) was 12.3 and 12.8 months in the CRT and consolidation treatment groups ( $p=0.840$ and 0.808$)$, respectively. Consolidation chemotherapy after concurrent CRT experiences has neither OS nor PFS survival benefit, compared with concurrent CRT alone. Thus, our study did not support favourable consolidation chemotherapy in patients with LA-NSCLC.
\end{abstract}

Keywords: Locally advanced non-small cell lung cancer, Consolidation chemotherapy, Concurrent chemoradiotherapy, Overall survival, Progression-free survival

\section{INTRODUCTION}

Lung cancer is the leading cause of cancer-related deaths worldwide and accounts for $18.4 \%$ of total cancer mortalities. ${ }^{1}$ According to the GLOBOCAN survey involving 185 countries, the global burden of the disease was $\sim 2.1$ million new lung cancers and 1.76 million deaths. ${ }^{1}$

Non-small cell lung cancer (NSCLC) represents about $80-85 \%$ of all newly diagnosed lung cancer cases. ${ }^{2}$ NSCLC is a lethal heterogeneous condition with a five-year survival rate ranging from 50\% in pathological stage I to $2 \%$ in stage IV. ${ }^{2,3}$ Approximately one-third of all NSCLC patients are diagnosed with stage III locally advanced NSCLC disease, and only a minority of these cases are considered operable. ${ }^{4}$ While recent advances in diagnostic and therapeutic agents have improved the management of the advanced disease, limited progress has been achieved in the diagnosis and treatment of early and locally advanced NSCLC (LA-NSCLC) disease stages. ${ }^{5}$

The management options for patients with stage III NSCLC include a combined modality therapy approach, such as combinations of surgery, chemotherapy and radiotherapy. Chemotherapy can be administered to patients before radiation (defined as sequential) or during radiation (defined as concurrent). Treatment can be applied as inductionconcurrent (chemotherapy before and during radiation) or concurrent consolidation (chemotherapy during and after radiotherapy). ${ }^{6}$ 
The standard treatment for patients with grade III LA-NSCLC is platinum-based chemotherapy followed by active surveillance with CRT, which provides improved overall survival (OS), as compared with sequential CRT or radiotherapy alone. ${ }^{7}$ A meta-analysis that included 19 randomised trials in 2010 showed an $8 \%$ reduction in mortality with CRT, as compared with that with single modality radiotherapy and a $13 \%$ reduction compared with sequential CRT. ${ }^{8}$ However, the outcome of CRT in patients with grade III NSCLC is still not encouraging, with 5-year OS rates ranging between 15\%$25 \% .^{9}$ The addition of consolidation chemotherapy has emerged as a potential treatment strategy, and many studies have focused on investigating the feasibility and effectiveness of this treatment following concurrent CRT administration in terms of possible improvements in PFS and OS. ${ }^{4,10}$ However, the efficacy of consolidation chemotherapy after concurrent CRT in patients with locally advanced NSCLC remains controversial. Given the lack of a significant benefit of survival for LA-NSCLC patients in a randomised phase III trial ${ }^{11}$ and observed pooled analysis from 45 studies $^{12}$, the definitive role of consolidation chemotherapy is unknown.

We aimed to evaluate the efficacy of platinumbased consolidation chemotherapy following concurrent CRT treatment and compare outcomes with concurrent CRT alone therapy in terms of their effects on PFS and OS in patients with locally advanced NSCLC.

\section{MATERIALS AND METHODS}

\section{Patient Selection}

This research was a single-centre retrospective study and was carried out from October 2009March 2018 as a cohort study at our hospital. A total of 136 patients diagnosed with unresectable locally advanced NSCLC who had received concurrent CRT with or without consolidation chemotherapy were included in the study. The participants were categorised into two groups based on whether they received consolidation chemotherapy or concurrent CRT.

All patients were histologically or cytologically proven to have NSCLC, diagnosed as stage III in accordance with the staging system of the American Joint Committee on Cancer in 2009 and Tumour Nodes Metastasis (TNM) classification. ${ }^{13}$ The diagnosis of the unresectable stage III LA-NSCLC disease was confirmed in most cases via up chest radiography, abdominal and thoracic computed tomography (CT) scans, brain magnetic resonance imaging to eradicate the presence of metastasis, bronchoscopy, endobronchial ultrasonography and FDG-positron emission tomography of the lungs. Clinical and demographic data of patients, including age, gender, smoking history, physical performance status, types of chemotherapy combination, dose reduction, the date of progression and last outpatient control or the date of exitus, were obtained from the hospital records.

\section{Study Design}

The patients were categorised into two groups and evaluated according to the administered treatment schedule. The first group was the consolidation group who received consolidation chemotherapy after concurrent CRT, and the second group comprised those who received concurrent CRT alone. In the consolidation group, in addition to prior CRT treatment, carboplatin plus paclitaxel or cisplatin plus etoposide were given as two cycles every 21 days. The records of the follow-up evaluations of all patients were used to obtain physical examination findings, complete blood cell counts (CBC), serum biochemistry, tumour markers, thoracic and abdominal CT scans and positron emission tomography for the suspicion of metastasis during 2 months after treatment.

The clinical and imaging examinations were performed every 3 months for the first year, and every 6 months in the following years. During the concurrent CRT period, CBC and serum biochemistry examinations were performed once a week. First response assessment was classified in three groups: partial response, stable disease or progressive disease according to Response Evaluation Criteria in Solid Tumors (RECIST version 1.1$)^{14}$, as follows: Partial response (PR) was defined as at least 30\% reduction from baseline in the longest diameters of target lesions. Progressive disease (PD) was defined as at least a $20 \%$ increase from baseline in 
the sum of longest diameters of target lesions or the appearance of one or more new lesions. Stable disease was defined as neither adequate lesion shrinkage to qualify for PR nor sufficient increase to qualify for PD. Relapses were recorded as local relapse or distant metastasis, and determined, as follows: Local relapse was defined as primary tumour recurrence or regional recurrence. Distant metastases were characterised by contralateral lung metastatic lymph nodes in the neck or axilla or metastasis to any other organ. If local relapse and distant metastasis were detected simultaneously, it was named 'Local and Distant Relapse', as shown Table 1. Disease progression was ascertained based on cytohistological or radiological examinations. Adverse effects were recorded via the electronic health information system of the hospital.

\section{Statistical Analysis}

The statistical analysis was performed using the SPSS software, version 18.0 (SPSS Inc. Chicago, IL, USA). Descriptive statistics were reported as percentages and medians. The demographic and clinical characteristics of patients were presented as medians and frequencies. OS was described as the time from diagnosis until death or last followup. PFS was described as the time from treatment initiation to disease progression or death. Progression was defined as local recurrence and/or distant metastasis. The groups were compared in terms of PFS and OS. Besides, the groups were compared according to age, gender, smoking duration, presence of dose reduction, response assessment, chemoregimens with concurrent radiotherapy and types of recurrence with local or distant metastasis. Categorical variables were analysed using chi-square or Fisher's exact test. Survival curves were obtained, and survival rates were determined using the Kaplan-Meier method, and comparisons were made with the log-rank test. Significant factors were identified in univariate analyses and were entered into a backward selection Cox regression model to determine independent predictors of survival. Univariate and multivariate analyses using a Cox proportional hazards (HZs) model was performed to assess potential prognostic factors for OS and PFS. A p-value of 0.05 or lower (p value< 0.05 ) was considered statistically significant.

\section{RESULTS}

A total of 136 patients with grade III NSCLC were included retrospectively in the study. The median age of the patients was 63 years (range: 42-82 years) and mostly male $(n=120,88.2 \%)$. In pathological examinations, the patients were diagnosed with various types of lung cancer, including squamous cell cancer (SCC), adenocarcinoma, not otherwise specified and large cell carcinoma $(63.2 \%$, $30.9 \%, 5.1 \%$ and $0.7 \%$, respectively). Precisely, 74 $(54.4 \%)$ patients were in stage IIIA and 62 (45.6\%) patients in stage IIIB. The Eastern Cooperative Oncology Group performance status (ECOG PS) was utilised to determine functional status; that is, the patients' ability to tolerate therapy under the severe disease condition. We found that the vast majority of patients' positive responses (95.6\%). Their mean smoking status was 41.2 packets/year.

Among the 136 patients, 77 patients (56.6\%) received consolidation chemotherapy after concurrent CRT, while 59 (43.4\%) patients were treated with concurrent CRT alone. A combination of treatments, consisting of cisplatin plus etoposide or carboplatin plus paclitaxel were used in the majority of participants, as the chemotherapy regimen $(\mathrm{n}=$ 77) and 16 of the 77 cases were administered with cisplatin as a single agent with concurrent CRT. All patients were administered curative thoracic radiotherapy with a minimum dose of 60 Gy radiation. For the concurrent CRT regimen, 68 (50\%) of the patients were treated with carboplatin plus paclitaxel, $50(36.8 \%)$ patients were administered cisplatin plus etoposide and 18 (13.2\%) patients received other platinum-containing regimens, including weekly cisplatin, cisplatin plus vinorelbine and cisplatin plus pemetrexed. Dose reduction was performed in $18(13.2 \%)$ patients due to adverse effects, including mucositis, renal toxicity, thrombocytopenia and neutropenia. No significant difference was detected between groups in the sense of dose reduction $(\mathrm{p}=0.139)$. After concurrent CRT, the consolidation group $(n=77)$ received two cycles of cisplatin- or carboplatin-based consolidation chemotherapy. The assessment of post-treatment responses showed local relapse in 42 patients (30.8\%), distant metastasis in 16 patients $(11.7 \%)$ and both in 9 patients $(6.6 \%)$. Metastasis localisations were as follows: brain $(8.1 \%)$, bone $(8 \%)$, 
International Journal of Hematology and Oncology

\begin{tabular}{|c|c|c|c|c|}
\hline & & C-CRT & CC-CRT & $p$-value \\
\hline \multirow[t]{2}{*}{ Gender } & Male & 52 (88.1\%) & 68 (88.3\%) & 0.975 \\
\hline & Female & 7 (11.9\%) & $9(11.7 \%)$ & \\
\hline \multicolumn{2}{|l|}{ Age (median) } & 71.1 & 66.4 & 0.496 \\
\hline \multirow[t]{2}{*}{ Smoking Status } & $\leq 40 \mathrm{pc} / \mathrm{y}$ & 25 (45.5\%) & 30 (39\%) & 0.285 \\
\hline & $>40 \mathrm{pc} / \mathrm{y}$ & 30 (54.6\%) & 47 (61\%) & 0.110 \\
\hline ECOG PS & $\leq 1$ & 56 (94.9\%) & 76 (98.7\%) & \\
\hline \multirow[t]{2}{*}{ Stage } & III a & 40 (69.0\%) & 34 (43.6\%) & $0.003^{*}$ \\
\hline & III b & 18 (31.0\%) & 44 (56.4\%) & \\
\hline \multirow[t]{2}{*}{ Histological type } & SCC & 41 (69.5\%) & 45 (55.4\%) & 0.298 \\
\hline & Non-SCC & $18(30.5 \%)$ & 32 (41.6\%) & \\
\hline \multirow[t]{2}{*}{ Dose reduction } & Yes & $4(6.9 \%)$ & 14 (18.2\%) & 0.056 \\
\hline & No & 54 (93.1\%) & 63 (81.8\%) & \\
\hline \multirow[t]{2}{*}{ Chemotherapy } & Cisplatin-based & 34 (57.6\%) & 26 (33.8\%) & $0.005^{\star}$ \\
\hline & Carboplatin-based & 25 (42.4\%) & $51(66.2 \%)$ & \\
\hline \multirow[t]{2}{*}{ Response } & PR at least & 28 (47.5\%) & 45 (58.4\%) & 0.394 \\
\hline & Non-PR & 21 (35.6\%) & 27 (35.1\%) & \\
\hline \multirow[t]{3}{*}{ Relapse } & Local relapse & 22 (73.3\%) & 20 (40\%) & $0.009^{\star}$ \\
\hline & Distant metastasis & $6(20 \%)$ & 10 (20\%) & \\
\hline & Local and distant & 1 (3.1\%) & $8(16 \%)$ & \\
\hline
\end{tabular}

pleura $(3.6 \%)$, liver $(2.2 \%)$ and adrenal gland $(2.2 \%)$.

Patient characteristics are shown in Table 1. No significant differences were found between the concurrent CRT only group and the consolidation treatment group in terms of gender, age, smoking status, histological subtype and ECOG PS performance status ( $p>0.05$ for all). Carboplatin-based chemotherapy was used more frequently than cisplatin-based chemotherapy in the consolidation treatment group $(\mathrm{p}=0.005)$. Before the treatment, 44 patients were in stage IIIB in the consolidation group, while this number was 18 in the CRT only group $(\mathrm{p}=0.004)$.

Partial and complete response, named at least PR due to less of CR, only two patients, in Table 1, was observed in 28 patients $(47.5 \%)$ in the CRT group and in 45 patients $(58.4 \%)$ in the consolidation chemotherapy group $(\mathrm{p}<0.001)$. In the CRT group, 22 patients $(73.3 \%$ ) had a local relapse, 6 patients (20\%) had distant metastasis, while 1 patient $(3.1 \%)$ had both local and distant metastasis. Post-treatment responses in the consolidation group revealed local relapse in 20 patients $(40 \%)$, distant metastasis in 10 patients $(20 \%)$ and both in 8 patients $(16 \%)(\mathrm{p}=0.009)$.

The median OS and PFS for all patients were 25.2 months and 12.8 months, respectively. The median OS was 27 months (range: 17.6-36.3) in the CRT group and 23.6 months (range: 18.2-29) in the consolidation treatment group $(\mathrm{p}=0.840)$. Median PFS was 12.3 months (range: 7.7-16.9) in the CRT group and 12.8 months (range: $9.7-16$ ) in the consolidation chemotherapy group $(\mathrm{p}=0.808)$. Therefore, no significant differences were found between the two groups in terms of PFS or OS (Figures 1 and 2).

The univariate analysis of prognostic factors for OS and PFS are shown in Table 2. We utilised this analysis to identify that having $<40$ packets/ 


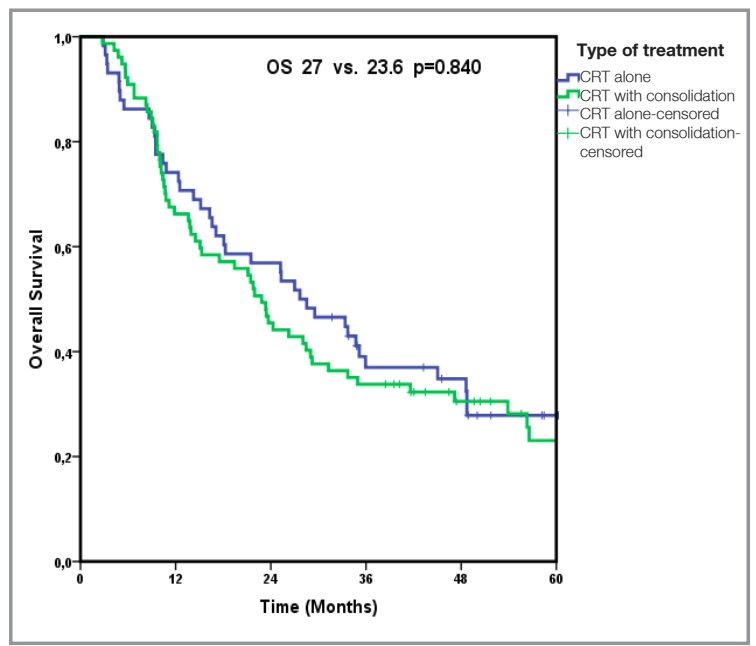

Figure 1. Kaplan-Meier curves of OS

year of smoking $(\mathrm{p}=0.025)$ and partial remission $(p<0.001)$ were significant prognostic factors for PFS and OS. Following the univariate analyses, smoking status and treatment response were evaluated in multivariate analyses, which showed that smoking of $<40$ packets/year and positive treatment response were two potential prognostic factors for survival. The mortality risk ratio of patients without PR was 2.1 (Hazard ratio range: 1.3-3.5, $\mathrm{p}=0.001$ ). Also, patients with PR (at least) were found to have statistically significant prolonged PFS (HR: 1.6, range: 1.0-2.6, $\mathrm{p}=0.038$ ). Smoking $<40$ packets/years was also associated with longer PFS (HR: 1.7, range: 1.0-2.8, $\mathrm{p}=0.028$ ). However, when the smoking status was evaluated in multivariate analysis for OS, we found no significant relationship (Table 3).

In consideration of adverse side effects, nausea $(\mathrm{n}=$ $104,76.4 \%)$, vomiting $(n=32,23.5 \%)$, fatigue $(n=$ $110,80.8 \%)$, mucositis $(n=39,28.6 \%)$, stomatitis $(n=26,19.1 \%)$, neuropathy $(n=37,27.2 \%)$, nephrotoxicity $(\mathrm{n}=7,5.1 \%)$, hepatotoxicity $(\mathrm{n}=8,5.8 \%)$, neutropenia $(n=53,38.9 \%)$, thrombocytopenia $(n=$ $13,9.5 \%)$ and anaemia $(n=34,25 \%)$ were seen. Due to a lack of side effects timing of the patients, comparable analyses were not performed between the consolidation and the CRT alone groups. However, side effects were evaluated due to carboplatin and cisplatin-based chemotherapy. As shown in Table 4, significant differences were detected for nausea, vomiting, fatigue, mucositis, stomatitis

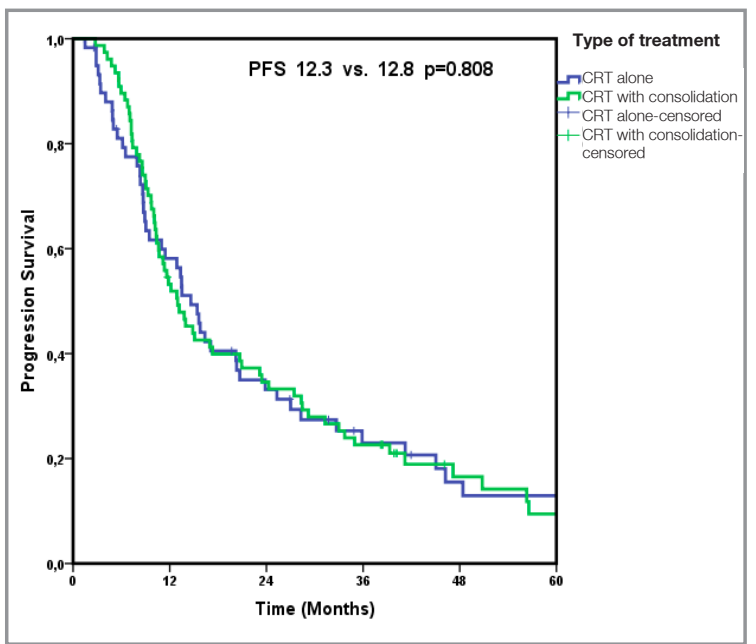

Figure 2. Kaplan-Meier curves of PFS

and neuropathy in carboplatin and cisplatin-based chemotherapy groups regardless of consolidation. We could not perform analysis regarding toxicity grade due to prolonged insufficient recorded dates on patients' files.

\section{DISCUSSION}

The current study aimed to assess the impact of consolidation chemotherapy on PFS and OS in patients with LA-NSCLC, known to have a wide range of clinical presentations, rendering the disease rather heterogeneous. ${ }^{15}$ The standard treatment for patients with inoperable stage III LA-NSCLC is concurrent chemoradiotherapy, which appears to enable local control of recurrence, decrease the development of distant metastasis and prolong survival. ${ }^{11,16}$ However, LA-NSCLC disease has a poor prognosis, and median survival times are only 1223.2 months. $^{9}$

Concurrent CRT plays a role as a radiosensitiser for the primary tumour area; however, due to dose management to avoid compromising disease control, the dosage might be insufficient for therapeutic performance for effective disease treatment. ${ }^{17}$ Therefore, it has been reported consistently that consolidation chemotherapy regimens might have different effects on the systemic disease, depending on several factors, including the number of cycles administered, chemotherapy regimens and 


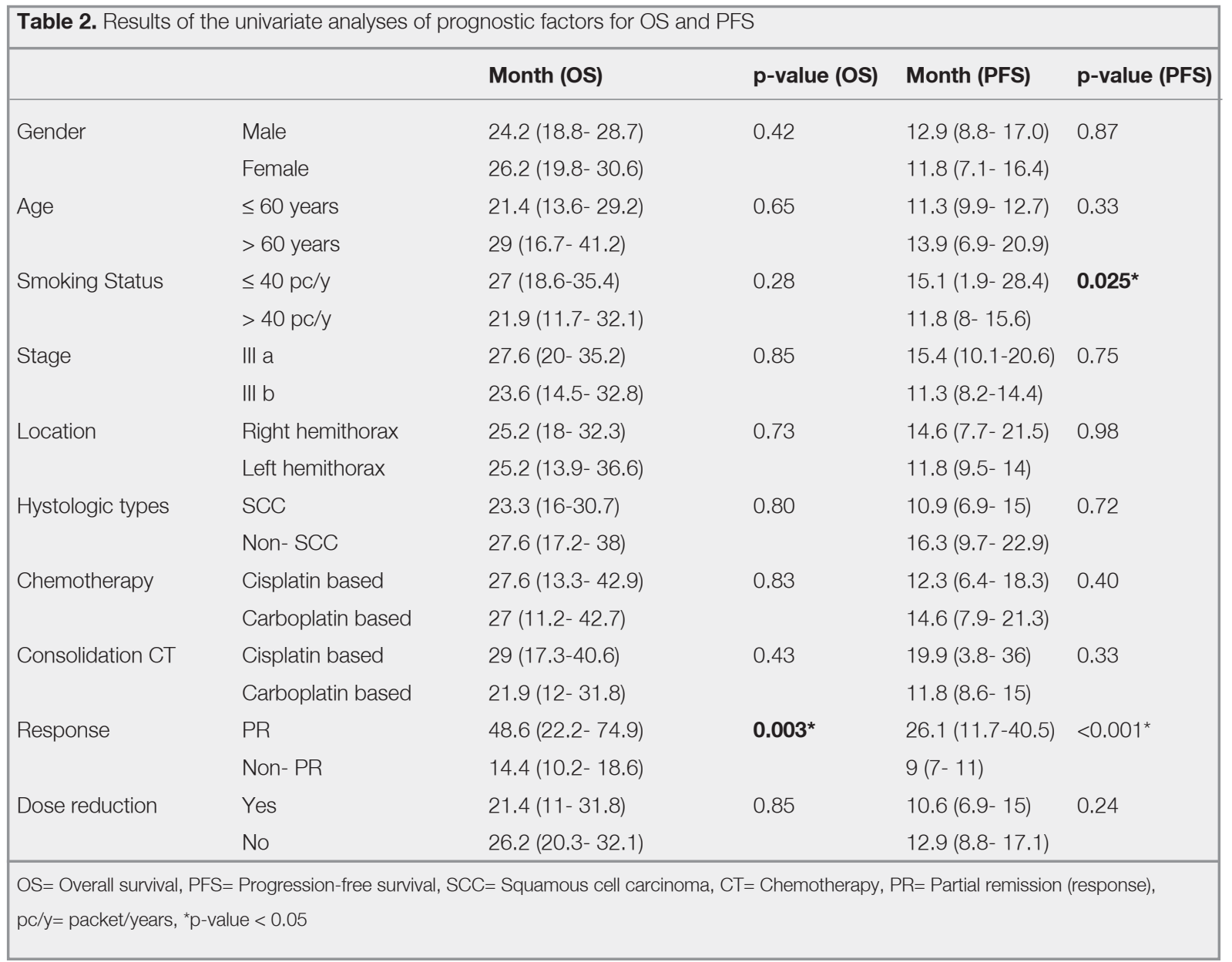

pre-consolidation therapies. ${ }^{16-18}$ However, currently, study outcomes with added consolidation CRT, seem to be inconclusive and somewhat controversial in many aspects. Hanna et al. ${ }^{11}$ demonstrated that there were no differences between PFS and OS in a randomised phase III clinical trial with 73 patients who were assigned to the docetaxel-consolidation group ( 3 cycles) and 74 patients who were enrolled as an observational group (both after concurrent CRT with cisplatin etoposide). Consistent with our results, we did not find any prolonged survival with a similar number of patient participants and we performed two cycles of consolidation therapy with various regimens in our study. Similarly, a multinational, randomised phase III study, involving 419 patients with grade III NSCLC conducted by Park et al. ${ }^{19}$ showed that consolidation therapy with three cycles of docetaxel-cisplatin failed to improve PFS in comparison with the observation group. These outcomes are again consistent with our findings. Further, Jalal and colleagues ${ }^{20}$, in their phase III trial for LA-NSCLC with or without consolidation therapy after CRT, revealed that additional docetaxel therapy provided no benefit for survival, but rather, rendered higher rates of toxicity and hospitalisation. A pooled analysis of the literature, including 34 phase II studies and 7 phase III studies conducted by Tsujino et al. ${ }^{12} \mathrm{dem}$ onstrated that consolidation chemotherapy after CRT failed to yield a favourable survival outcome or risk reduction in the mortality of patients with LA-NSCLC.

In our study, we found differences between our two groups for the stage, choice of platin-based treatment and type of relapse, while the outcomes of the OS and PFS were similar in both groups. We noticed conflicting results available in the literature: Mutlu et al. ${ }^{21}$ showed in 74 inoperable patients with locally advanced NSCLC that consolidation treatment had better outcomes concerning OS and 
International Journal of Hematology and Oncology

Table 3. Results of the multivariate analyses of prognostic factors for OS and PFS

\begin{tabular}{|c|c|c|c|c|}
\hline & PFS HR (95\% Cl) & p-value (PFS) & OS HR (95\% Cl) & p-value (OS) \\
\hline \multicolumn{5}{|l|}{ Smoking time } \\
\hline$\leq 40 \mathrm{pc} / \mathrm{y}$ & Reference & & Reference & \\
\hline$>40 \mathrm{pc} / \mathrm{y}$ & $1.7(1-2.8)$ & $0.028^{\star}$ & $1.1(0.7-1.8)$ & 0.411 \\
\hline \multicolumn{5}{|l|}{ Response } \\
\hline PR (at least) & Reference & & Reference & \\
\hline Non-PR & $1.6(1-2.6)$ & $0.038^{\star}$ & $2.1(1.3-3.5)$ & $0.001^{*}$ \\
\hline
\end{tabular}

$\mathrm{OS}=$ Overall survival; $\mathrm{PFS}=$ Progression-free survival; $\mathrm{HR}=\mathrm{Hazard}$ ratio; $\mathrm{Cl}=$ Confidence interval; $\mathrm{PR}=\mathrm{Partial}$ response

PFS, compared with induction chemotherapy, followed by CRT or CRT alone. In their study, all the patients were in stage IIIB with a TNM classification of T4N2M0, which might explain the results, as these patients might need more systemic chemotherapy to eradicate micrometastases and circulating tumour cells; thus, this could possibly cause an alteration in the efficacy of additional therapy.
Wang et $\mathrm{al}^{4}{ }^{4}$, in a meta-analysis, including five separate studies, reported that the treatment of LANSCLC with CRT, followed by consolidation therapy, improved OS, but not PFS. In another study, Liu et al. ${ }^{18}$ demonstrated in a retrospective study, with 113 patients' inclusion who received consolidation therapy for at least two cycles and 90 patients who received CRT alone, that consolida-

Table 4. Differences in the side effects of platin-based chemotherapies regardless of consolidation

\begin{tabular}{|c|c|c|c|c|}
\hline Side effect & & Cisplatin-based (\%) & Carboplatin-based (\%) & p-value $\left({ }^{*}<0.05\right)$ \\
\hline \multirow[t]{2}{*}{ Nausea } & Yes & $54(91.5)$ & $50(64.9)$ & $<0.001^{*}$ \\
\hline & No & $5(8.5)$ & $27(35.1)$ & \\
\hline \multirow[t]{2}{*}{ Vomiting } & Yes & $20(33.8)$ & $12(15.5)$ & $0.012^{*}$ \\
\hline & No & $39(66.2)$ & $65(84.5)$ & \\
\hline \multirow[t]{2}{*}{ Fatigue } & Yes & $56(95)$ & $53(68.9)$ & $<0.001^{*}$ \\
\hline & No & $3(5)$ & $24(31.1)$ & \\
\hline \multirow[t]{2}{*}{ Mucositis } & Yes & $26(44)$ & $13(16.8)$ & $0.001^{*}$ \\
\hline & No & $33(56)$ & 64 (83.2) & \\
\hline \multirow[t]{2}{*}{ Stomatitis } & Yes & $17(28.8)$ & $9(11.6)$ & $0.011^{*}$ \\
\hline & No & $42(71.2)$ & $68(88.4)$ & \\
\hline \multirow[t]{2}{*}{ Neuropathy } & Yes & $6(10.1)$ & $31(40.2)$ & $<0.001^{\star}$ \\
\hline & No & $53(89.9)$ & $46(59.8)$ & \\
\hline \multirow[t]{2}{*}{ Nephrotoxicity } & Yes & $3(5)$ & $4(5.1)$ & 0.166 \\
\hline & No & $56(95)$ & 73 (94.9) & \\
\hline \multirow[t]{2}{*}{ Hepatotoxicity } & Yes & $3(5)$ & $5(6.9)$ & 0.566 \\
\hline & No & $56(95)$ & $72(93.1)$ & \\
\hline \multirow[t]{2}{*}{ Neutropenia } & Yes & $23(38.9)$ & $30(38.9)$ & 0.569 \\
\hline & No & $36(61.1)$ & $47(61.1)$ & \\
\hline \multirow[t]{2}{*}{ Thrombocytopenia } & Yes & $7(11.8)$ & $6(7.7)$ & 0.304 \\
\hline & No & $52(88.2)$ & 71 (92.3) & \\
\hline \multirow[t]{2}{*}{ Anaemia } & Yes & $16(27.1)$ & 18 (23.3) & 0.381 \\
\hline & No & 43 (72.9) & 59 (76.7) & \\
\hline
\end{tabular}


tion treatment had significantly better survival than CRT alone (median OS: 27 months vs. 16 months; 5-year OS: $30.4 \%$ vs. $22.5 \%, \mathrm{p}=0.012$ ) without increased toxicity. Moreover, they showed better survival advantages with consolidation chemotherapy after concurrent CRT mostly in the following groups of patients: those aged $<60$ years, males, those with non-squamous histology, patients with stage IIIB disease, those that showed treatment response and recipients of radiotherapy dose $\geq 60$ Gy. ${ }^{18}$

Prognostic factors are critical in the evaluation of disease progression and treatment modalities. The widely accepted prognostic factors for LA-NSCLC include female gender, stage IIIA, weight loss and good performance status. ${ }^{18,22}$ In our study, we found that treatment response and smoking status are significant prognostic factors for PFS and OS. We revealed that patients with PR had 1.6 times longer PFS and 2.1 times longer OS than patients without PR. Our results support the view of Jeremic and colleagues ${ }^{23}$, who suggested that patients with complete or PR were more likely to benefit from consolidation therapy. We also observed that smoking cigarettes $<40$ packets/year associated with 1.7 times longer PFS than smoking $>40$ packets/year. The PFS disadvantage of high smoking history, as a prognostic factor, might be relevant co-morbidities. ${ }^{24}$ However, any data of comorbidity was recorded.

Further, our study found a difference between the two groups in terms of the cancer stage. More stage IIIA patients were identified in the CRT group, whereas stage IIIB was more common in the consolidation group. Thus, we expected this observation to make a difference between the groups in univariate analysis; however, the CRT group might not have been able to display prolonged survival due to the absence of consolidation chemotherapy. Tsujino et al. ${ }^{12}$ aimed to evaluate whether consolidation chemotherapy, after concurrent CRT, is beneficial based on the same goal as our group. They published a pooled analysis from the literature, and, interestingly, found no difference between the consolidation group and the CRT group in terms of stage III and IIIB disease, presenting $<33 \%$ of the ratio in stage IIIA group having shorter OS. Contrary to our study, which failed to show an appreci- able difference, possibly due to the smaller number of study participants.

Although the optimal chemoregimen in the treatment of LA-NSCLC could not be selected, platin-based chemotherapies are preferred to radiosensitising agents. ${ }^{15}$ According to our literature review, and in our study in which cisplatin-based and carboplatin-based chemotherapies were chosen, carboplatin-based treatment, in particular, was more noticeable in our consolidation group than the concurrent CRT group. Ezer et al..$^{25}$ showed that carboplatin-based CRT displayed a parallel survival association with lower rates of toxicities. However, we did not notice any survival difference in our carboplatin-based group, which is in agreement with the indicated study.

Our present study does not preclude limitations. First, because of the nature of the retrospective study design, our analyses do not represent homogeneous data towing to no constant treatment choice at the centre. This situation could obstruct the achievement of more meaningful results. Second, our study included heterogeneous consolidation regimens, unequal treatment arms and heterogeneous radiotherapy dosages (despite defining a threshold of at least $60 \mathrm{~Gy}$ ), likely to affect our conclusions. Third, we examined patients from a single centre with similar ethnicity (Turkish population). Thus, patient characteristics, including ethnicity, might have played a significant role in survival and response rate to chemotherapy in lung cancer. $^{26}$

\section{Conclusion}

In conclusion, we found no significant differences between the CRT only group and the consolidation treatment group regarding PFS and OS. Additionally, our results demonstrated that patients with grade III NSCLC treated by consolidation chemotherapy after prior concurrent CRT experiences have no survival benefit, compared with those treated with concurrent CRT alone. Further, we showed that smoking status and treatment response were prognostic factors for PFS and OS. Therefore, clinical studies with novel treatment approaches, including molecular-the utilisation of targeted agents and innovative immunotherapy 
strategies are necessary to establish the feasibility of consolidation chemotherapy regimens.

\section{REFERENCES}

1. Bray F, Ferlay J, Soerjomataram I, et al. Global cancer statistics 2018: GLOBOCAN estimates of incidence and mortality worldwide for 36 cancers in 185 countries. CA Cancer $\mathrm{J}$ Clin 68: 394-424, 2018.

2. Reck M, Rabe KF. Precision Diagnosis and Treatment for Advanced Non-Small-Cell Lung Cancer. N Engl J Med 377: 849-861, 2017.

3. Shlomi D, Ben-Avi R, Balmor GR, et al. Screening for lung cancer: time for large-scale screening by chest computed tomography. Eur Respir J 44: 217-238, 2014.

4. Wang $X$, Ding $X$, Kong D, et al. The effect of consolidation chemotherapy after concurrent chemoradiotherapy on the survival of patients with locally advanced non-small cell lung cancer: a meta-analysis. Int J Clin Oncol 22: 229-236, 2017.

5. Reich JM. A critical appraisal of overdiagnosis: estimates of its magnitude and implications for lung cancer screening. Thorax 63: 377-383, 2008.

6. Duma N, Santana-Davila R, Molina JR. Non-Small Cell Lung Cancer: Epidemiology, Screening, Diagnosis, and Treatment. Mayo Clin Proc 94: 1623-1640, 2019.

7. Yoon SM, Shaikh T, Hallman M. Therapeutic management options for stage III non-small cell lung cancer. World J Clin Oncol 8: 1, 2017.

8. O'Rourke N, Figuls MRI, Bernadó NF, Macbeth F. Concurrent chemoradiotherapy in non-small cell lung cancer. Cochrane Database of Systematic Reviews, 2010.

9. Ramnath N, Dilling TJ, Harris LJ, et al. Treatment of stage III non-small cell lung cancer: diagnosis and management of lung cancer: American College of Chest Physicians evidencebased clinical practice guidelines. Chest 143: e314S-e340S, 2013.

10. Urvay S, Yücel B. Induction or consolidation chemotherapy for unresectable stage III non-small-cell lung cancer patients treated with concurrent chemoradiation: a retrospective study. Ortadogu Medical Journal/Ortadogu Tip Dergisi 11: 40-46, 2019.

11. Hanna N, Neubauer M, Yiannoutsos C, et al. Phase III study of cisplatin, etoposide, and concurrent chest radiation with or without consolidation docetaxel in patients with inoperable stage III non-small-cell lung cancer: The Hoosier Oncology Group and US Oncology. J Clin Oncol 26: 5755-5760, 2008.

12. Tsujino $\mathrm{K}$, Kurata $\mathrm{T}$, Yamamoto $\mathrm{S}$, et al. Is consolidation chemotherapy after concurrent chemo-radiotherapy beneficial for patients with locally advanced non-small-cell lung cancer?: A pooled analysis of the literature. J Thorac Oncol 8: 1181-1189, 2013.
13. Goldstraw P, Crowley J, Chanksy K, et al. Lung Cancer Staging Project: Proposals for the Revision of the TNM Stage Groupings in the Forthcoming (Seventh) Edition of the TNM Classification of Malignant Tumors. J Thorac Oncol 2: 706714, 2007. The above article ran in the August 2007 issue without showing the figures on page 711 in color. We regret any inconvenience this may have caused. In this issue, we present Figures 2 and 3 from that article in full color. $J$ Thorac Oncol 2: 985, 2007.

14. Therasse P, Eisenhauer E, Verweij J. RECIST revisited: a review of validation studies on tumour assessment. Eur $\mathrm{J}$ Cancer 42:1031-1039, 2006.

15. Filippi AR, Di Muzio J, Badellino S, et al. Locally-advanced non-small cell lung cancer: shall immunotherapy be a new chance? J Thorac Dis 10: S1461, 2018.

16. Eroglu C, Orhan O, Unal D, et al. Concomitant chemoradiotherapy with docetaxel and cisplatin followed by consolidation chemotherapy in locally advanced unresectable nonsmall cell lung cancer. Ann Thorac Med 8: 109, 2013.

17. Harris JP, Patel MI, Loo BW, et al. A population-based comparative effectiveness study of chemoradiation regimens and sequences in stage III non-small cell lung cancer. Lung Cancer 108: 173-182, 2017.

18. Liu L, Bi N, Ji Z, et al. Consolidation chemotherapy may improve survival for patients with locally advanced non-smallcell lung cancer receiving concurrent chemoradiotherapyretrospective analysis of 203 cases. BMC Cancer 15: 715, 2015.

19. Ahn JS, Ahn YC, Kim JH, et al. Multinational randomized phase III trial with or without consolidation chemotherapy using docetaxel and cisplatin after concurrent chemoradiation in Inoperable stage III non-small-cell lung cancer: KCSG-LU05-04. J Clin Oncol 33: 2660-2666, 2015.

20. Jalal S, Riggs H, Melnyk A, et al. Updated survival and outcomes for older adults with inoperable stage III non-small-cell lung cancer treated with cisplatin, etoposide, and concurrent chest radiation with or without consolidation docetaxel: analysis of a phase III trial from the Hoosier Oncology Group (HOG) and US Oncology. Ann Oncol 23: 1730-1738, 2012.

21. Mutlu $\mathrm{H}$, Arslan $D$, Gündüz $S$, et al. The optimal treatment modality in patients with T4N2M0 non-small cell lung cancer: the best choice may be definitive chemoradiotherapy followed by consolidation chemotherapy. Chemotherapy 60: 107-111, 2014.

22. Stanley KE. Prognostic factors for survival in patients with inoperable lung cancer. J Natl Cancer Inst 65: 25-32, 1980.

23. Jeremic B. Consolidation chemotherapy after concurrent radiochemotherapy in locally advanced non-small cell lung cancer may have been beneficial if we only knew where it have worked. J Thorac Oncol 9: e7, 2014.

24. Agata E, Adam A. Comorbidities in lung cancer. Pneumonol Alergol Pol 84: 186-192, 2016 
International Journal of Hematology and Oncology

25. Ezer N, Smith CB, Galsky MD, et al. Cisplatin vs. carboplatinbased chemoradiotherapy in patients $>65$ years of age with stage III non-small cell lung cancer. Radiother Oncol 112: 272-278, 2014.

26. Soo RA, Kawaguchi T, Loh M, et al. Differences in outcome and toxicity between Asian and caucasian patients with lung cancer treated with systemic therapy. Future Oncology 8: 451-462, 2012.

\section{Correspondence:}

\section{Dr. Fatma Bugdayci BASAL}

Dr. Abdurrahman Yurtaslan Onkoloji Egitim ve

Arastirma Hastanesi

Tibbi Onkoloji Bolumu

Yenimahalle, ANKARA / TURKEY

Tel: (+90-505) 7714036

e-mail: dr.fatmabb@gmail.com

\section{ORCIDs:}

Fatma Bugdayci Basal

0000-0003-3562-2670

Ferit Aslan

0000-0002-9153-6921

Guliz Zengin

0000-0002-9575-8214

Irem Bilgetekin

0000-0003-1154-5850

Omur Berna Oksuzoglu

0000-0002-2756-8646

Umut Demirci

0000-0002-4833-6721 